\title{
Prediction of 5-year overall survival in cervical cancer patients treated with radical hysterectomy using computational intelligence methods
}

\author{
Bogdan Obrzut ${ }^{1}$, Maciej Kusy ${ }^{2 *}$, Andrzej Semczuk ${ }^{3}$, Marzanna Obrzut ${ }^{4}$ and Jacek Kluska ${ }^{2}$
}

\begin{abstract}
Background: Computational intelligence methods, including non-linear classification algorithms, can be used in medical research and practice as a decision making tool. This study aimed to evaluate the usefulness of artificial intelligence models for 5-year overall survival prediction in patients with cervical cancer treated by radical hysterectomy.

Methods: The data set was collected from 102 patients with cervical cancer FIGO stage IA2-IIB, that underwent primary surgical treatment. Twenty-three demographic, tumor-related parameters and selected perioperative data of each patient were collected. The simulations involved six computational intelligence methods: the probabilistic neural network (PNN), multilayer perceptron network, gene expression programming classifier, support vector machines algorithm, radial basis function neural network and k-Means algorithm. The prediction ability of the models was determined based on the accuracy, sensitivity, specificity, as well as the area under the receiver operating characteristic curve. The results of the computational intelligence methods were compared with the results of linear regression analysis as a reference model.
\end{abstract}

Results: The best results were obtained by the PNN model. This neural network provided very high prediction ability with an accuracy of 0.892 and sensitivity of 0.975 . The area under the receiver operating characteristics curve of PNN was also high, 0.818 . The outcomes obtained by other classifiers were markedly worse.

Conclusions: The PNN model is an effective tool for predicting 5-year overall survival in cervical cancer patients treated with radical hysterectomy.

Keywords: Cervical cancer, 5-year overall survival, Computational intelligence methods, Probabilistic neural network

\section{Background}

Cervical cancer is the fourth most common malignancy among females worldwide [1]. Approximately 527,600 new cases are reported annually all over the world and 265,700 women died in 2012 [1]. In Poland, the annual incidence of invasive cervical cancer is $8.9 / 100,000$ woman, and in 2012, 2783 new cases of cervical cancer were diagnosed and 1669 women died [2].

\footnotetext{
*Correspondence: mkusy@prz.edu.pl

${ }^{2}$ Faculty of Electrical and Computer Engineering, Rzeszow University of Technology, al. Powstancow Warszawy 12, 35-959 Rzeszow, Poland

Full list of author information is available at the end of the article
}

The prediction of clinical outcome is a key element of the therapeutic decision-making process. For cervical cancer, the established platform for planning the treatment is the International Federation of Gynecology and Obstetrics (FIGO) classification. Although the FIGO staging system serves as the main tool for estimating the general prognosis, it does not include all established prognostic factors, such as lymph node metastases, lymph-vascular space invasion, deep stromal infiltration, or histologic subtype. Another method for individual prediction of survival in cervical carcinoma is the recently developed nomograms based on selected demographic and clinical parameters $[3,4]$. None of these systems take 
into account intra/postoperative complications and concomitant diseases, however, which could alter patients outcome [5].

The aim of the present study was to develop a universal model for predicting overall survival in individual patients with cervical cancer, based on the demographic characteristics, tumor-related parameters, and selected perioperative data. We used computational intelligence methods, that have been widely applied in oncology [6-10]. Artificial neural networks as advanced computer programs enable the discovery of complex relations within data sets, that cannot be detected with conventional linear statistical analysis. Computational intelligence methods to predict overall survival have not yet been applied for patients with cervical cancer treated by radical hysterectomy.

\section{Methods}

This study originally included 117 patients with cervical cancer (FIGO stages IA2-IIB) treated at the Department of Obstetrics and Gynaecology of the Rzeszow State Hospital in Poland between 1998 and 2001. The preoperative diagnosis was based on the histopathologic examination of tissue material obtained from cervical biopsy and fractionated abrasion. In disputable cases (15 patients), cold knife cervical conization was performed.

All patients underwent radical hysterectomy class III and pelvic lymphadenectomy. Before the surgery, basic laboratory tests, electrocardiograms, and anaesthesiology consultations were performed. Perioperative prophylactic antibiotics and thromboembolic prophylaxis, were applied for each patient. Laparotomy was performed through a vertical midline or low transverse incision, depending on the local conditions. Intraoperative and postoperative complications were recorded and classified prospectively according to the classification proposed by Chassagne et al. [11].

After the period of postoperative recovery, all patients were forwarded to the Department of Gynecological Oncology of the Rzeszow State Hospital. Some of the patients received adjuvant radiotherapy. The qualifying criteria were as follows: presence of metastases to the lymph nodes, lymph-vascular space invasion, or the presence of neoplastic tissue within the surgical incision and non-squamous types of cervical cancer. Radiotherapy was administered in the following manner: teletherapy (50 Gy to the area of pelvis minor in 25 fractions of 2 Gy; BOX technique) and brachytherapy (2 fractions of lowdose rate; total dose 30 Gy). During the study period, chemotherapy was not routinely applied.

Follow-up was conducted once a month during the first year after the operation, every 3 months during the second year of observation, twice annually for 3 to 5 years in the Oncology Outpatient Clinic and the Department of Gynaecological Oncology at the Rzeszow State Hospital,
Rzeszow, Poland. The 5-year follow-up data from all the subjects were used to validate examined computational intelligence models designed for the prediction of death within 60 months.

Data available at the time of discharge, derived from histopathologic examination of the surgical specimen and obtained during the follow-up, were collected. In total, 23 variables were identified, including 4 demographic characteristics: age, BMI, hormonal status, presence of concomitant diseases; 13 tumor-related parameters: FIGO stage, histologic type, grade, tumor size $\leq 4 \mathrm{~cm}$ or $>4 \mathrm{~cm}$, lymph nodes status, number of lymph nodes dissected, number of positive lymph nodes, lymph node ratio (ratio of positive to totally removed lymph nodes), lymph-vascular space invasion, surgical margins status, parametrial involvement, deep stromal invasion (outer $1 / 3$ of the cervical stroma), postoperative radiotherapy; 6 selected perioperative variables: surgery time, median blood lost, presence of intraoperative complications, presence of postoperative complications, type of complications, and length of hospital stay. The above listed variables are presented in Table 1 . To present continuous values, the median measure (along with variable range) was used.

These variables were used in the simulations, which included six clasiffiers: the probabilistic neural network (PNN), multilayer perceptron network (MLP), gene expression programming classifier (GEP), support vector machines algorithm (SVM), radial basis function neural network (RBFNN) and k-Means method. All considered models were simulated in DTREG software [12].

PNN is a feedforward neural network created by Specht [13]. PNN is composed of the input layer represented by the variables of the input vector, the pattern layer and the summation layer consisting of $G$ neurons where each one computes the signal only for patterns that belong to $g$ th class. The output layer of the network determines the label for a classified vector in accordance with Bayes's decision rule based on all the summation layer neuron signals. The performance of PNN can be optimized by selecting the form of the smoothing parameter $(s p)$ used for activation of neurons in the pattern layer.

MLP is a feedforward neural network [14]. This network is composed of an input layer, hidden layers, and an output layer. The number of hidden layers, the optimal number of neurons in hidden layers and the appropriate activation functions must be determined for this model.

GEP is an emulating biological evolution algorithm, that creates and evolves computer programs [15]. The programs are encoded by chromosomes composed of the genes. Within the population, evolution is performed by computing the expression of each chromosome, applying predefined genetic operators and calculating the fitness. 
Table 1 Demographic characteristics and clinicopathologic data in the study group

\begin{tabular}{ll}
\hline Number of patients & 102 \\
\hline Median age & $46(29-73)$ \\
Median BMI $\left[\mathrm{kg} / \mathrm{m}^{2}\right]$ & $25.1(17.5-45.0)$
\end{tabular}

Hormonal status

$\begin{array}{ll}\text { Premenopausal } & 71 \\ \text { Postmenopausal } & 31\end{array}$

Concomitant diseases

$\begin{array}{lr}\text { Hypertension } & 21 \\ \text { Diabetes mellitus } & 3 \\ \text { Ischaemic heart diseaase } & 6 \\ \text { Others } & 3\end{array}$

FIGO stage

$\begin{array}{ll}\mid \mathrm{A} 2 & 15 \\ \mathrm{IB} 1 & 51 \\ \mathrm{IB} 2 & 8 \\ \mathrm{IA} & 7 \\ \mathrm{IB} & 21\end{array}$

Histologic type

\begin{tabular}{|c|c|}
\hline & Squamous \\
\hline & Non-squamous \\
\hline Grading & \\
\hline & G1 \\
\hline & G2 \\
\hline & G3 \\
\hline
\end{tabular}

Median surgery time [min]

Median blood lost $(\triangle \mathrm{Hb})[\mathrm{g} \%]$

Tumour size $[\mathrm{cm}]$

$\begin{array}{ll}\leq 4 & 69 \\ >4 & 33\end{array}$

Median number of removed lymph nodes

33

$13(1-40)$

Lymph nodes status

$\begin{array}{ll}\text { Negative } & 77 \\ \text { Positive } & 25\end{array}$

Median number of positive lymph nodes

Median lymph node ratio

Lymph-vascular space invasion

$$
\text { Absent }
$$

Present

Deep stromal invasion

$\begin{array}{ll}\text { Absent } & 66 \\ \text { Present } & 36\end{array}$

Parametrium infiltration

Absent 78

Table 1 Demographic characteristics and clinicopathologic data in the study group (Continued)

\begin{tabular}{lll}
\hline Number of patients & 102 \\
\hline Surgical margins status & Negative & 89 \\
& Positive & 13 \\
Intraoperative complications & & 5 \\
Postoperative complications & & 42 \\
Type of complications & Mild & 38 \\
& Moderate & 2 \\
& Severe & 7 \\
Median hospital stay [days] & & $12(5-49)$ \\
Postoperative radiotherapy & & 57 \\
& Yes & 45 \\
\hline
\end{tabular}

SVM is the classification algorithm proposed by Vapnik [16]. The SVM algorithm requires solving the quadratic programming optimization problem. For the SVM model, various kernel functions and their parameters need to be explored. Furthermore, the model's capacity control parameter $C$ must be selected.

RBFNN, similar to PNN and MLP, is a feedforward neural network [17]. This model consists of three layers: an input layer, a radial basis hidden layer, and a linear output layer. The number of neurons in the hidden layer and the parameters of the RBFNN training method must be found [18].

The k-Means clustering algorithm partitions input data into $k$ clusters and provides a center of each cluster [19]. As a result, the records within each cluster are similar to each other and distinct from records in other clusters. The predictions for the unknown cases are made by assigning them the category of the nearest cluster center.

The prediction ability of the models was determined based on the accuracy (Acc), sensitivity (Sen), specificity (Spe), and the area under the receiver operating characteristic curve (AUROC). The above parameters were obtained using a 10-fold cross validation procedure [20]. The simulations were conducted 20 times, preserving a random selection of subsets. The results were averaged and the standard deviation was computed. As a reference model, we applied the logistic regression analysis, which is widely used in medical research [10, 21, 22].

\section{Statistical analysis}

The AUROC value of particular classifiers and logistic regression model were compared using pairwise T-tests. Differences were considered statistically significant when 
$p<0.05$. All statistical analysis were performed using MathWorks' Matlab R2012a software.

\section{Results}

Among 117 patients that qualified for a radical Piver III hysterectomy and pelvic lymphadenectomy, 15 did not enter the trial: 3 were excluded because the histopathologic analysis of the operative specimen revealed an endometrial cancer with cervical extension, 4 continued postoperative treatment and follow-up at another institution, 3 refused further participation in the study protocol, and 5 were lost from follow-up. The remaining 102 were considered eligible and were enrolled in the study.

The median patients' age was 46 years (range, 29-73). Thirty-one patients were postmenopausal. The median BMI was $25.1 \mathrm{~kg} / \mathrm{m}^{2}$ (range, 17.5-45.0). Concomitant diseases were reported in 33 women (Table 1). The prevailing type was squamous-cell carcinoma (89.2\%).

The length of surgery ranged between 80 and $310 \mathrm{~min}$ (median 190). The median blood loss, measured by a decrease in the hemoglobin level, was $\triangle \mathrm{Hb}=3.9 \mathrm{~g} \%$ (range, 0.3-7.8).

The median number of dissected lymph nodes was 13 (range, 1-40). Positive lymph nodes were diagnosed in 25 patients. The lymph node ratio ranged from 0 to 1 . Parametrial involvement was observed in 24 patients, and deep stromal invasion was identified in 36 cases.

Lymph-vascular space invasion was observed in 19 patients. In 13 cases, positive surgical margins were reported. The median hospital stay, calculated from the date of surgery to the day of discharge, was 12 days (range, 5-49).

The number of perioperative complications was 47 (46.1\%). Intraoperative complications occurred in 5 patients. Postoperative complications, counted to 30 days after surgery, occured in 42 patients. The vast majority of cases were mild or medium degree complications that did not constitute threats to the health or life of the patients. Severe perioperative complications (pulmonary embolism, bleeding from the inferior vena cava, rupture of duodenal ulcer, genitourinary fistulas) occurred in 7 patients.

Median follow-up period in the study group was 51.7 months (range, 6-60 months). During the follow-up, recurrence was identified in 23 patients (22.6\%). Pelvic recurrence was detected in 13 patients. The remaining 10 subjects were diagnosed with distant metastases. The patients' status at last observation was as follows: alive79 , cancer-related death-23. The overall 5-year survival was $77.5 \%$ in the study group.

The best results in the prediction of 5-year overall survival in cervical cancer patients treated with radical hysterectomy were obtained by PNN. This model enabled the prediction of the 5-year overall survival with the highest accuracy (0.892), sensitivity (0.975), and specificity (0.609). The MLP and GEP also showed high accuracy ( 0.802 and 0.800 , respectively) and sensitivity $(\approx 0.93)$, but markedly lower specificity. The AUROC for PNN (0.818) also significantly surpassed the values of this parameter for the remaining classifiers (Fig. 1). The averaged accuracy, sensitivity, specificity and AUROC value obtained for all applied computational intelligence methods and linear regression model are presented in Table 2 .

Because PNN provided the highest values of all measured parameters (Table 2), we determined the confusion matrix for this model. As presented in Table 3, of 23 cases of patient death, 9 were incorrectly predicted. On the other hand, only two cases were misclassified among patients who survived.

\section{Discussion}

PNN as well as other computational intelligence methods have been applied to various medical classification tasks $[9,10,23]$. For example, to detect arrhythmia based on digital processing of electrocardiograms [24], for bleeding detection in wireless capsule endoscopy [25], as well as for estimating the risk of mortality after cardiac surgery [26]. In recent years, artificial neural networks have also been used in our studies to predict complications in cervical cancer patients treated by radical hysterectomy $[7,8]$. To the best of our knowledge (Pubmed search database), this is the first study to apply computational intelligence methods for prediction of survival in cervical cancer patients treated with radical hysterectomy.

Application of the PNN, which utilized 23 variables (demographic characteristics, tumor-related parameters, and selective perioperative data), enabled the prediction of the 5-year overall survival with an accuracy of 0.892 in cervical cancer patients (Table 2). This model revealed high sensitivity (0.975), although the specificity was lower (0.609). This may result from much lower number of death cases in the study group (class imbalance). Our results are comparable to similar reports in which neural networks were applied for outcome prediction of cancer patients. For example, an artificial neural network was able to predict survival in colorectal cancer patients with an overall accuracy of $90 \%$ [6]. The predictive AUROC of PNN model for 5-year survival in esophageal carcinoma was 0.884 [9]. In another study, the ANN model enabled the precise prediction of mortality after primary liver carcinoma with accuracy and AUROC equal to 0.973 and 0.840 , respectively [10].

The variables used in the simulations are mostly wellestablished prognostic factors in uterine cervical cancer. Of these variables, one of the most important is the clinical stage of the disease, as demonstrated in the 1990s $[27,28]$. Although the FIGO clinical staging is imperfect, 


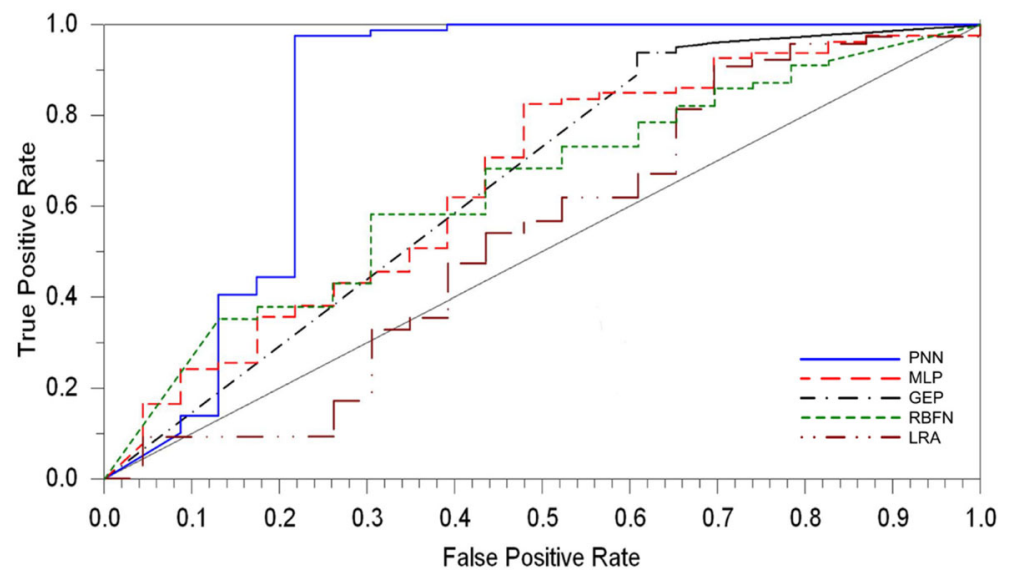

Fig. 1 The receiver operating characteristic curves. Plots are shown for the models with AUROC $>0.5$

survival rates of patients with cervical cancer correlate with the FIGO stage of neoplasm progression [29].

The correlation between tumor size and prognosis for patients with cervical cancer has also been widely investigated over several decades [30-36]. Some researchers have concluded that tumor size in cervical cancer has an independent prognostic value, regardless of age, ethnicity, histologic grade, or even the type of treatment [37].

Similarly, another known prognostic factor is the depth of stromal invasion of the uterine cervix and infiltration of the parametrium [38-42].

Despite several studies, there is no conclusive evidence regarding the impact of the histologic subtype of cervical cancer on survival rates [43]. While some studies identified no significant differences between cervical squamous cell carcinoma and adenocarcinoma [44-48], others reported unfavorable outcomes for patients with uterine cervix adenocarcinoma [49-53].

Another controversial prognostic factor in patients with cervical cancer is the histologic grade. Some studies indicate that poorly-differentiated squamous cell cervical carcinoma has an unfavorable prognosis [54],

Table 2 The accuracy, sensitivity, specificity and the area under receiver operating characteristic curve obtained for the set of 23 variables

\begin{tabular}{llllll}
\hline & Acc & Sen & Spe & \multicolumn{2}{l}{ AUROC } \\
\hline PNN & 0.892 & 0.975 & 0.609 & 0.818 & $p<0.001$ \\
MLP & 0.802 & 0.937 & 0.339 & 0.659 & $p<0.001$ \\
GEP & 0.800 & 0.930 & 0.352 & 0.651 & $p<0.001$ \\
SVM & 0.740 & 0.956 & 0.000 & 0.478 & $p<0.001$ \\
LRA & 0.703 & 0.804 & 0.357 & 0.559 & Non applicable \\
RBFNN & 0.693 & 0.780 & 0.396 & 0.640 & $p<0.001$ \\
k-Means & 0.611 & 0.757 & 0.109 & 0.406 & $p<0.001$ \\
\hline
\end{tabular}

whereas another studies did not confirm these observations $[55,56]$.

Several studies report a close relationship between patient survival and lymph node involvement [57-61]. Moreover, the number of positive lymph nodes is a more accurate prognostic factor than the presence of metastases [62-65]. Attention has recently been focused on another parameter - the lymph node ratio [3].

Factors associated with a higher risk of cancer recurrence include the lymph-vascular space invasion (LVSI). This reflects, in part, the high correlation between LVSI and involvement of the pelvic lymph nodes, yet numerous studies suggest that this parameter is an independent prognostic factor $[66,67]$.

Published findings related to the potential significance of patient age as a prognostic factor in cervical cancer are contradictory. In several studies, no differences in the survival rates among patients in different age groups were identified [68-71]. Other studies, however, suggest that the prognosis is significantly worse in younger patients [72-74].

A positive surgical margin is considered an important risk factor in the recurrence of cervical cancer. Lee et al. observed a significantly worse disease-free survival and overall survival in patients with positive margins, but only in the univariate analysis [75]. Multivariate analysis showed no significant impact of positive surgical margins on the prognosis. Similar observations were also reported by Landoni et al. [76].

Table 3 Confusion matrix for the PNN model

\begin{tabular}{lll}
\hline \multirow{2}{*}{ Actual outcome } & \multicolumn{2}{l}{ Predicted outcome } \\
\cline { 2 - 3 } & Died & Survived \\
\hline Died & 14 & 9 \\
Survived & 2 & 77
\end{tabular}


To date, there is no evidence that the surgery time or length of hospital stay have a direct impact on survival. These parameters are related to the course of the operation and convalescence, and can increase due, for example, to complications. Similarly, perioperative complications in themselves do not influence the natural history of carcinoma, but their treatment may delay adjuvant therapy, which in turn, can reduce the probability of survival. Concomitant diseases, reflecting general patient health condition, may also constrain adjuvant therapy and in consequence negatively influence the prognosis [5].

Finally, we used all the listed variables in the simulations because, on one hand, the accuracy of artificial neural networks could be improved by increasing the number of factors [77], and, on the other hand, even non-significant variables must have a non-zero effect on survival [3].

Only a few papers in the literature deal with prediction of survival in cervical cancer patients. Ochi et al. applied artificial neural networks for survival prediction in patients with uterine cervical cancer treated by radiotherapy using different data sets [78]. The highest AUROC value of this model was 0.778 . Polterauer et al. developed a nomogram based on six variables: FIGO stage (IB-IV), tumor size $(\leq 2 \mathrm{~cm}$ vs $>2 \mathrm{~cm}$ ), age, histologic subtype, lymph node ratio, and parametrial involvement [3]. The value of the c-index, which is conceptually similar to receiver operating characteristics curve analysis $[79,80]$, was 0.723 . The most recent study by Zhou et al. establishes a nomogram predicting 5-year overall survival of surgically-treated stage IA-IIB cervical cancer patients [4]. The authors used a number of metastatic lymph nodes, lymph-vascular space invasion, stromal invasion, parametrial invasion, tumor diameter, and histology as input variables. The c-index of this model was 0.71 . A precise comparison of our results with the results presented by others is difficult because of differences in the study population, data set and methodology. Nevertheless, the AUROC value of one of our predictive models (PNN) outperformed the values obtained in the above-mentioned studies.

The presented study has a few limitations. First, the number of patients was too small to enable detailed analysis of the impact of histologic type on survival in the non-squamous cell carcinoma. The group also included adenocarcinomas as well as cases of glandularsquamous, microcellular, and undifferentiated cervical carcinomas. For simulations, we used clinical data from patients treated surgically in 1998-2001. In subsequent years, standards of treatment for cervical cancer were significantly modified. While in past decades, surgery for patients in FIGO stage IIB was quite common, it is currently performed only in highly-specialized centers [81-85]. Moreover, concurrent chemo-radiotherapy has been introduced on a large scale. In this context, the presented results refer to only a selected group of patients.

The current study has also several strengths. The main advantage is its design. As previously mentioned, based on a Pubmed database search, this is the first study to apply computational intelligence methods to predict overall survival in cervical cancer patients treated by radical hysterectomy. Moreover, the study group of patients came entirely from a single institution, where the same principles of diagnostic and surgical procedures were carefully applied. This increases the strength of our study, due to its consistency and uniformity.

\section{Conclusions}

Computational intelligence methods enable credible survival prediction for cervical cancer patients. The prediction ability of the PNN measured by the AUROC value outperformed that of the MLP, GEP and RBFNN and the linear regression model. The low AUROC value for the SVM algorithm and k-Means method disqualified them as predictive classifiers. We conclude that $\mathrm{PNN}$ is a very effective tool for predicting 5-year overall survival in cervical cancer patients treated with radical hysterectomy.

\section{Abbreviations}

Acc: Accuracy; AUROC: Area under receiver operating characteristic curve; BMI: Body mass index; FIGO: The International federation of gynecology and obstetrics; GEP: Gene expression programming; MLP: Multilayer perceptron; PNN: Probabilistic neural network; RBFNN: Radial basis function neural network; Sen: Sensitivity; Spe: Specificity; SVM: Support vector machines

\section{Acknowledgments}

There are no acknowledgments related to this study.

\section{Funding}

The study was granted by Lublin Medical University, Lublin, Poland (grant $326 / 17$ to $A S)$.

\section{Availability of data and materials}

All the clinical data used in this study are stored in the Rzeszow State Hospital archives. The informed consent which the participants signed does not presuppose sharing the data in publicly available repositories.

\section{Authors' contributions}

BO co-initiated and co-led the study design, collected the data, co-led the data analysis and interpretation, co-wrote the first draft of the paper, coordinated draft revisions and co-wrote the final manuscript. MK co-initiated and co-led the study design, co-led the data analysis and interpretation, co-wrote the first draft of the paper and co-wrote the final manuscript. AS contributed to the data analysis and interpretation, and draft revisions. $\mathrm{MO}$ contributed to data collection and interpretation. JK contributed to the study conception and design, data analysis and interpretation, and draft revisions. All authors read and approved the final manuscript.

\section{Ethics approval and consent to participate}

This research is involved with human participants and is approved by the Bioethics Committee of the Regional Medical Chamber (reg. no. 3/98; 20/02/1998)

\section{Consent for publication}

Each patient participated in the current study under the 'Ethics, consent and permissions' heading based on Bioethics Committee approval. This manuscript does not include an individual participant's data in any form (including images, videos, voice recordings etc). 


\section{Competing interests}

The authors declare that they have no competing interests.

\section{Publisher's Note}

Springer Nature remains neutral with regard to jurisdictional claims in published maps and institutional affiliations.

\section{Author details}

${ }^{1}$ Department of Gynaecology and Obstetrics, Faculty of Medicine, University of Rzeszow, Lwowska 60, 35-301 Rzeszow, Poland. ${ }^{2}$ Faculty of Electrical and Computer Engineering, Rzeszow University of Technology, al. Powstancow Warszawy 12, 35-959 Rzeszow, Poland. ${ }^{3}$ IIND Department of Gynecology, Lublin Medical University, al. Raclawickie 1, 20-059 Lublin, Poland. ${ }^{4}$ Faculty of Medicine, University of Rzeszow, al. Kopisto 2a, 35-959 Rzeszow, Poland.

\section{Received: 5 July 2017 Accepted: 21 November 2017}

Published online: 12 December 2017

\section{References}

1. Torre LA, Bray F, Siegel RL, Ferlay J, Lortet-Tieulent J, Jemal A. Global cancer statistics, 2012. CA: Cancer J Clin. 2015;65(2):87-108.

2. Wojciechowska UZW, Didkowska J. Cancer in Poland. 2013. Available from: http://www.onkologia.org.pl. Accessed 15 Feb 2016.

3. Polterauer S, Grimm C, Hofstetter G, Concin N, Natter C, Sturdza A, et al Nomogram prediction for overall survival of patients diagnosed with cervical cancer. British J Cancer. 2012;107(6):918-24.

4. Zhou H, Li X, Zhang Y, Jia Y, Hu T, Yang R, et al. Establishing a Nomogram for Stage IA-IIB Cervical Cancer Patients after Complete Resection. Asian Pacific J Cancer Prevent. 2015:16(9):3773-7.

5. Brewer N, Borman B, Sarfati D, Jeffreys M, Fleming ST, Cheng S, et al. Does comorbidity explain the ethnic inequalities in cervical cancer survival in New Zealand? A retrospective cohort study. BMC Cancer. 2011;11(1):132.

6. Bottaci L, Drew PJ, Hartley JE, Hadfield MB, Farouk R, Lee PW, et al. Artificial neural networks applied to outcome prediction for colorectal cancer patients in separate institutions. The Lancet. 1997;350(9076): 469-72.

7. Kluska J, Kusy M, Obrzut B. Prediction of Radical Hysterectomy Complications for Cervical Cancer Using Computational Intelligence Methods. In: Rutkowski L, Korytkowski M, Sherer R, Tadeusiewicz R, Zadeh LA, Zurada J, editors. Artificial Intelligence and Soft Computing: 11th International Conference, ICAISC 2012, Zakopane, Poland, April 29-May 3, 2012, Proceedings, Part II. Berlin: Springer-Verlag. 2012. p. 259-67.

8. Kusy M, Obrzut B, Kluska J. Application of gene expression programming and neural networks to predict adverse events of radical hysterectomy in cervical cancer patients. Med Biol Eng Comput. 2013;51(12):1357-65.

9. Sato F, Shimada Y, Selaru FM, Shibata D, Maeda M, Watanabe G, et al. Prediction of survival in patients with esophageal carcinoma using artificial neural networks. Cancer. 2005;103(8):1596-605.

10. Shi HY, Lee KT, Lee HH, Ho WH, Sun DP, Wang JJ, et al. Comparison of artificial neural network and logistic regression models for predicting in-hospital mortality after primary liver cancer surgery. PloS ONE. 2012;7(4):1-6

11. Chassagne D, Sismondi $P$, Horiot J, Sinistrero G, Bey $P$, Zola P, et al. A glossary for reporting complications of treatment in gynecological cancers. Radiothe Oncol. 1993;26(3):195-202.

12. Sherrod PH. DTREG Predictive Modelling Software. Available from: http://www.dtreg.com. Accessed 17 May 2017.

13. Specht DF. Probabilistic neural networks and the polynomial Adaline as complementary techniques for classification. Neural Netw IEEE Trans. 1990;1(1):111-21.

14. Rumelhart DE, McClelland JL, PDP Research Group C, (eds). Parallel Distributed Processing: Explorations in the Microstructure of Cognition, Vol. 1: Foundations. Cambridge: MIT Press; 1986.

15. Ferreira C. Gene expression programming: mathematical modeling by an artificial intelligence: Springer: Berlin; 2006

16. Vapnik $V$. The nature of statistical learning theory. Springer-Verlag, New York; 1995.
17. Broomhead DS, Lowe D. Multivariable functional interpolation and adaptive networks. Complex Systems. 1988;2:321-55.

18. Chen S, Wang $X$, Harris CJ. Experiments with repeating weighted boosting search for optimization signal processing applications. Syst Man Cybernet Part B Cybernet IEEE Trans. 2005;35(4):682-93.

19. Hartigan JA, Wong MA. A k-means clustering algorithm. J R Stat Soc Series C (Appl Stat). 1979;1:100-8

20. Kohavi R. A study of cross-validation and bootstrap for accuracy estimation and model selection. In: Proceedings of International Join Conference on Artificial Intelligence, Vol. 14. Stanford: Morgan Kaufmann. 1995. p. 1137-43.

21. Biglarian A, Bakhshi E, Gohari MR, Khodabakhshi R. Artificial neural network for prediction of distant metastasis in colorectal cancer. Asian Pac J Cancer Prevent. 2012;13(3):927-30.

22. Chien CW, Lee YC, Ma T, Lee TS, Lin YC, Wang W, et al. The application of artificial neural networks and decision tree model in predicting post-operative complication for gastric cancer patients. Hepatogastroenterology. 2008;55(84):1140-5.

23. Zhang XP, Wang ZL, Tang L, Sun YS, Cao K, Gao Y. Support vector machine model for diagnosis of lymph node metastasis in gastric cancer with multidetector computed tomography: a preliminary study. BMC Cancer. 2011;11(1):10

24. Haseena HH, Mathew AT, Paul JK. Fuzzy clustered probabilistic and mult layered feed forward neural networks for electrocardiogram arrhythmia classification. J Med Syst. 2011;35(2):179-88.

25. Pan G, Yan G, Qiu X, Cui J. Bleeding detection in wireless capsule endoscopy based on probabilistic neural network. J Med Syst. 2011;35(6): $1477-84$

26. Orr RK. Use of a probabilistic neural network to estimate the risk of mortality after cardiac surgery. Med Decis Making. 1997;17(2):178-85.

27. Stehman FB, Bundy BN, Disaia PJ, Keys HM, Larson JE, Fowler WC Carcinoma of the cervix treated with radiation therapy I. A multi-variate analysis of prognostic variables in the gynecologic oncology group. Cancer. 1991;67(11):2776-85.

28. Fyles AW, Pintilie M, Kirkbride P, Levin W, Manchul LA, Rawlings GA Prognostic factors in patients with cervix cancer treated by radiation therapy: results of a multiple regression analysis. Radiothe Oncol. 1995;35(2):107-17.

29. Quinn M, Benedet J, Odicino F, Maisonneuve P, Beller U, Creasman W, et al. Carcinoma of the cervix uteri. Int J Gynecol Obstet. 2006;95(S1): 43-103.

30. Piver MS, Chung WS. Prognostic significance of cervical lesion size and pelvic node metastases in cervical carcinoma. Obstet Gynaecol. 1975;46(5):507-10.

31. Baltzer J, Koepcke W. Tumor size and lymph node metastases in squamous cell carcinoma of the uterine cervix. Arch Gynecol Obstet. 1979;227(3):271-8

32. Perez CA, Grigsby PW, Chao KC, Mutch DG, Lockett MA. Tumor size irradiation dose, and long-term outcome of carcinoma of uterine cervix. Int J Radiat Oncol Biol Phys. 1998;41(2):307-17.

33. Horn LC, Fischer U, Raptis G, Bilek K, Hentschel B. Tumor size is of prognostic value in surgically treated FIGO stage II cervical cancer. Gynecol Oncol. 2007;107(2):310-5.

34. Eifel PJ, Jhingran A, Levenback CF, Tucker S. Predictive value of a proposed subclassification of stages I and || cervical cancer based on clinical tumor diameter. Int J Gynecol Cancer. 2009;19(1):2-7.

35. Kristensen GB, Abeler VM, Risberg B, Tropé C, Bryne M. Tumor size, depth of invasion, and grading of the invasive tumor front are the main prognostic factors in early squamous cell cervical carcinoma. Gynecol Oncol. 1999:74(2):245-51

36. Trattner M, Graf AH, Lax S, Forstner R, Dandachi N, Haas J, et al. Prognostic factors in surgically treated stage $\mathrm{lb}-\mathrm{llb}$ cervical carcinomas with special emphasis on the importance of tumor volume. Gynecol Oncol. 2001:82(1):11-16.

37. Wagner AE, Pappas L, Ghia AJ, Gaffney DK. Impact of tumor size on survival in cancer of the cervix and validation of stage IIA1 and IIA2 subdivisions. Gynecol Oncol. 2013;129(3):517-21.

38. Delgado G, Bundy B, Zaino R, Sevin BU, Creasman WT, Major F. Prospective surgical-pathological study of disease-free interval in patients with stage IB squamous cell carcinoma of the cervix: a Gynecologic Oncology Group study. Gynecol Oncol. 1990;38(3):352-7. 
39. Sedlis A, Bundy BN, Rotman MZ, Lentz SS, Muderspach LI, Zaino RJ. $A$ randomized trial of pelvic radiation therapy versus no further therapy in selected patients with stage IB carcinoma of the cervix after radical hysterectomy and pelvic lymphadenectomy: A Gynecol Oncol. Group Study. Gynecol Oncol. 1999;73(2):177-83.

40. Inoue T, Okumura M. Prognostic significance of parametrial extension in patients with cervical carcinoma stages IB, IIA, and IIB. A study of 628 cases treated by radical hysterectomy and lymphadenectomy with or without postoperative irradiation. Cancer. 1984;54(8):1714-9.

41. Zreik TG, Chambers JT, Chambers SK. Parametrial involvement, regardless of nodal status: a poor prognostic factor for cervical cancer Obstet Gynaecol. 1996;87(5):741-6.

42. Peters IIIWA, Liu P, Barrett RJ, Stock RJ, Monk BJ, Berek JS, et al. Concurrent chemotherapy and pelvic radiation therapy compared with pelvic radiation therapy alone as adjuvant therapy after radical surgery in high-risk early-stage cancer of the cervix. J Clin Oncol. 2000;18(8):1606-13.

43. Winer I, Alvarado-Cabrero I, Hassan O, Ahmed QF, Alosh B, Bandyopadhyay S, et al. The prognostic significance of histologic type in early stage cervical cancer-A multi-institutional study. Gynecol Oncol. 2015;137(3):474-8.

44. Shingleton HM, Bell MC, Fremgen A, Chmiel JS, Russell AH, Jones WB, et al. Is there really a difference in survival of women with squamous cell carcinoma, adenocarcinoma, and adenosquamous cell carcinoma of the cervix?. Cancer. 1995;76(S10):1948-55.

45. Look KY, Brunetto VL, Clarke-Pearson DL, Averette HE, Major FJ, Alvarez RD, et al. An analysis of cell type in patients with surgically staged stage IB carcinoma of the cervix: a Gynecol Oncol. Group study. Gynecol Oncol. 1996;63(3):304-11.

46. Kilgore LC, Soong SJ, Gore H, Shingleton HM, Hatch KD, Partridge EE. Analysis of prognostic features in adenocarcinoma of the cervix. Gynecol Oncol. 1988;31(1):137-48.

47. Alfsen GC, Kristensen GB, Skovlund E, Pettersen EO, Abeler VM. Histologic subtype has minor importance for overall survival in patients with adenocarcinoma of the uterine cervix: a population-based study of prognostic factors in 505 patients with non-squamous cell carcinomas of the cervix. Cancer. 2001;92(9):2471-83.

48. Lee KBM, Lee JM, Park CY, Lee KB, Cho HY, Ha SY. What is the difference between squamous cell carcinoma and adenocarcinoma of the cervix? A matched case-control study. Int J Gynecol Cancer. 2006;16(4):1569-73.

49. Chen RJ, Lin YH, Chen CA, Huang SC, Chow SN, Hsieh CY. Influence of histologic type and age on survival rates for invasive cervical carcinoma in Taiwan. Gynecol Oncol. 1999:73(2):184-90.

50. Nakanishi T, Ishikawa H, Suzuki Y, Inoue T, Nakamura S, Kuzuya K. A comparison of prognoses of pathologic stage lb adenocarcinoma and squamous cell carcinoma of the uterine cervix. Gynecol Oncol. 2000;79(2): 289-93.

51. Irie T, Kigawa J, Minagawa Y, Itamochi H, Sato S, Akeshima R, et al. Prognosis and clinicopathological characteristics of lb-llb adenocarcinoma of the uterine cervix in patients who have had radical hysterectomy. Eur J Surg Oncol. 2000;26(5):464-7.

52. Takeda N, Sakuragi N, Takeda M, Okamoto K, Kuwabara M, Negishi H, et al. Multivariate analysis of histopathologic prognostic factors for invasive cervical cancer treated with radical hysterectomy and systematic retroperitoneal lymphadenectomy. Acta Obstetricia et Gynecologica Scandinavica. 2002;81(12):1144-51.

53. Davy ML, Dodd TJ, Luke CG, Roder DM. Cervical cancer: effect of glandular cell type on prognosis, treatment, and survival. Obstet Gynaecol. 2003;101(1):38-45.

54. Landoni F, Maneo A, Cormio G, Perego P, Milani R, Caruso O, et al. Class II versus class III radical hysterectomy in stage IB-IIA cervical cancer: a prospective randomized study. Gynecol Oncol. 2001;80(1):3-12.

55. Van Nagell J, Donaldson E, Parker J, Van Dyke A, Wood E. The prognostic significance of pelvic lymph node morphology in carcinoma of the uterine cervix. Obstet Gynecol Surv. 1978;33(3):201-4.

56. Zaino R, Ward S, Delgado G, Bundy B, Gore H, Fetter G, et al. Histopathologic predictors of the behavior of surgically treated stage IB squamous cell carcinoma of the cervix a gynecologic oncology group study. Cancer. 1992;69(7):1750-8.

57. Burghardt E, Pickel H, Haas J, Lahousen M. Prognostic factors and operative treatment of stages IB to IIB cervical cancer. Am J Obstet Gynecol. 1987;156(4):988-96.
58. Creasman WT, Zaino RJ, Major FJ, DiSaia PJ, Hatch KD, Homesley HD. Early invasive carcinoma of the cervix (3 to $5 \mathrm{~mm}$ invasion): risk factors and prognosis: a Gynecol Oncol. Group study. Am J Obstet Gynecol. 1998;178(1):62-5.

59. Inoue T. Prognostic significance of the depth of invasion relating to nodal metastases, parametrial extension, and cell types: A study of 628 cases with stage IB, IIA, and IIB cervical carcinoma. Cancer. 1984;54(12): 3035-42.

60. Obrzut B, Semczuk A, Narog M, Obrzut M, Krol P. Prognostic parameters for patients with cervical cancer FIGO stages IA2-IIB: a long-term follow-up. Oncology. 2017;93(2):106-14.

61. Obrzut B. Impact of the extent of surgery on the treatment outcomes in patients with cervical cancer FIGO IA2-IIB. Poznan: Osrodek Wydawnictw Naukowych; 2008. (In Polish).

62. Lapuz C, Kondalsamy-Chennakesavan S, Bernshaw D, Khaw P, Narayan K. Stage IB cervix cancer with nodal involvement treated with primary surgery or primary radiotherapy: Patterns of failure and outcomes in a contemporary population. J Med Imaging Radiat Oncol. 2016;60: 274-82.

63. Inoue T, Morita K. The prognostic significance of number of positive nodes in cervical carcinoma stages IB, IIA, and IIB. Cancer. 1990;65(9): 1923-7.

64. Heller P, Malfetano J, Bundy B, Barnhill D, Okagaki T. Clinical-pathologic study of stage IIB, III, and IVA carcinoma of the cervix: extended diagnostic evaluation for paraaortic node metastasis-a Gynecol Oncol Group study. Gynecol Oncol. 1990;38(3):425-30.

65. Sakuragi N, Satoh C, Takeda N, Hareyama H, Takeda M, Yamamoto R, et al. Incidence and distribution pattern of pelvic and paraaortic lymph node metastasis in patients with stages IB, IIA, and IIB cervical carcinoma treated with radical hysterectomy. Cancer. 1999;85(7):1547-54.

66. Kamura T, Tsukamoto N, Tsuruchi N, Saito T, Matsuyama T, Akazawa K, et al. Multivariate analysis of the histopathologic prognostic factors of cervical cancer in patients undergoing radical hysterectomy. Cancer. 1992;69(1):181-6.

67. Kristensen GB, Abeler VM, Risberg B, Tropé C, Bryne M. Tumor size, depth of invasion, and grading of the invasive tumor front are the main prognostic factors in early squamous cell cervical carcinoma. Gynecol Oncol. 1999;74(2):245-51.

68. Baltzer J, Koepcke W, Lohe K, Ober K, Zander J. Age and 5-year survival rates in patients with operated carcinoma of the cervix. Gynecol Oncol. 1982;14(2):220-4.

69. Meanwell CA, Kelly KA, Wilson S, Roginski C, Woodman C, Griffiths R, et al. Young age as a prognostic factor in cervical cancer: analysis of population based data from 10022 cases. Br Med J (Clin Res Ed). 1988;296(6619):386-91.

70. Rutledge FN, Mitchell MF, Munsell M, Bass S, McGuffee V, Atkinson EN. Youth as a prognostic factor in carcinoma of the cervix: a matched analysis. Gynecol Oncol. 1992;44(2):123-30.

71. Austin J, Degefu S, Torres J, Bush DJ, O'quinn AG, Ozmen N, et al. Cervical carcinoma in women less than 35 years of age. South Med J. 1994;87(3):375-9.

72. Stuart G, Robertson D, Fedorkow D, Duggan M, Nation J. Recurrent and persistent squamous cell cervical carcinoma in women under age 35. Gynecol Oncol. 1988;30(2):163-72.

73. Fedorkow DM, Robertson DI, Duggan MA, Nation JG, McGregor SE, Stuart GC. Invasive squamous cell carcinoma of the cervix in women less than 35 years old: recurrent versus nonrecurrent disease. Am J Obstet Gynecol. 1988;158(2):307-11.

74. Dattoli MJ, Gretz HF, Beller U, Lerch IA, Demopoulos RI, Beckman EM, et al. Analysis of multiple prognostic factors in patients with stage IB cervical cancer: Age as a major determinant. Int J Radiat Oncol Biol Phys. 1989;17(1):41-7.

75. Lee YY, Choi CH, Kim TJ, Lee JW, Kim BG, Lee JH, et al. A comparison of pure adenocarcinoma and squamous cell carcinoma of the cervix after radical hysterectomy in stage IB-IIA. Gynecol Oncol. 2011;120(3):439-43.

76. Landoni F, Maneo A, Cormio G, Perego P, Milani R, Caruso O, et al. Class II versus class III radical hysterectomy in stage IB-IIA cervical cancer: a prospective randomized study. Gynecol Oncol. 2001;80(1):3-12.

77. Burke HB, Goodman PH, Rosen DB, Henson DE, Weinstein JN, Harrell FE, et al. Artificial neural networks improve the accuracy of cancer survival prediction. Cancer. 1997;79(4):857-62. 
78. Ochi T, Murase K, Fujii T, Kawamura M, Ikezoe J. Survival prediction using artificial neural networks in patients with uterine cervical cancer treated by radiation therapy alone. International J Clin Oncol. 2002;7(5):294-300.

79. Tripepi G, Jager KJ, Dekker FW, Zoccali C. Statistical methods for the assessment of prognostic biomarkers (Part I): discrimination. Nephrol Dial Transpl. 2010;25(5):1399-401.

80. Tripepi G, Jager KJ, Dekker FW, Zoccali C. Diagnostic methods 2: receiver operating characteristic (ROC) curves. Kidney Int. 2009;76(3):252-6.

81. Kasamatsu T, Onda T, Sawada M, Kato T, Ikeda Si. Radical hysterectomy for FIGO stage IIB cervical cancer: clinicopathological characteristics and prognostic evaluation. Gynecol Oncol. 2009;114(1):69-74.

82. Mabuchi S, Okazawa M, Matsuo K, Kawano M, Suzuki O, Miyatake T, et al. Impact of histological subtype on survival of patients with surgically-treated stage IA2-IIB cervical cancer: adenocarcinoma versus squamous cell carcinoma. Gynecol Oncol. 2012;127(1):114-20.

83. Chai Y, Wang T, Wang J, Yang Y, Gao Y, Gao J, et al. Radical hysterectomy with adjuvant radiotherapy versus radical radiotherapy for FIGO stage III cervical cancer. BMC Cancer. 2014;14(1):63.

84. Lee YJ, Kim DY, Lee SW, Park JY, Suh DS, Kim JH, et al. A postoperative scoring system for distant recurrence in node-positive cervical cancer patients after radical hysterectomy and pelvic lymph node dissection with para-aortic lymph node sampling or dissection. Gynecol Oncol. 2017:144(3):536-40

85. Kodama J, Seki N, Ojima Y, Nakamura K, Hongo A, Hiramatsu Y. Prognostic factors in node-positive patients with stage IB-IIB cervical cancer treated by radical hysterectomy and pelvic lymphadenectomy. Int J Gynecol Obstet. 2006;93(2):130-5.

\section{Submit your next manuscript to BioMed Central and we will help you at every step:}

- We accept pre-submission inquiries

- Our selector tool helps you to find the most relevant journal

- We provide round the clock customer support

- Convenient online submission

- Thorough peer review

- Inclusion in PubMed and all major indexing services

- Maximum visibility for your research

Submit your manuscript at www.biomedcentral.com/submit 